\title{
On the resistance matrix of a graph
}

\author{
Jiang Zhou* \\ College of Science \\ College of Computer Science and Technology \\ Harbin Engineering University \\ Harbin, PR China \\ zhoujiang04113112@163.com \\ Zhongyu Wang \\ School of Management \\ Harbin Institute of Technology \\ Harbin, PR China \\ wangzhy@hit.edu.cn

\section{Changjiang $\mathrm{Bu}$} \\ College of Science \\ Harbin Engineering University \\ Harbin, PR China \\ buchangjiang@hrbeu.edu.cn
}

Submitted: Jun 1, 2015; Accepted: Feb 16, 2016; Published: Mar 4, 2016

Mathematics Subject Classifications: 05C50, 05C12, 15A09

\begin{abstract}
Let $G$ be a connected graph of order $n$. The resistance matrix of $G$ is defined as $R_{G}=\left(r_{i j}(G)\right)_{n \times n}$, where $r_{i j}(G)$ is the resistance distance between two vertices $i$ and $j$ in $G$. Eigenvalues of $R_{G}$ are called R-eigenvalues of $G$. If all row sums of $R_{G}$ are equal, then $G$ is called resistance-regular. For any connected graph $G$, we show that $R_{G}$ determines the structure of $G$ up to isomorphism. Moreover, the structure of $G$ or the number of spanning trees of $G$ is determined by partial entries of $R_{G}$ under certain conditions. We give some characterizations of resistance-regular graphs and graphs with few distinct R-eigenvalues. For a connected regular graph $G$ with diameter at least 2 , we show that $G$ is strongly regular if and only if there exist $c_{1}, c_{2}$ such that $r_{i j}(G)=c_{1}$ for any adjacent vertices $i, j \in V(G)$, and $r_{i j}(G)=c_{2}$ for any non-adjacent vertices $i, j \in V(G)$.
\end{abstract}

Keywords: Resistance distance; Resistance matrix; Laplacian matrix; Resistanceregular graph; R-eigenvalue

\section{Introduction}

All graphs considered in this paper are simple and undirected. Let $V(G)$ and $E(G)$ denote the vertex set and the edge set of a graph $G$, respectively. The resistance distance is a

\footnotetext{
${ }^{*}$ Corresponding author.
} 
distance function on graphs introduced by Klein and Randić [19]. Let $G$ be a connected graph of order $n$. For two vertices $i, j$ in $G$, the resistance distance between $i$ and $j$, denoted by $r_{i j}(G)$, is defined to be the effective resistance between them when unit resistors are placed on every edge of $G$. The resistance matrix of $G$ is defined as $R_{G}=\left(r_{i j}(G)\right)_{n \times n}$. Eigenvalues of $R_{G}$ are called $R$-eigenvalues of $G$. The resistance (distance) in graphs has been studied extensively $[8,9,11,12,14,19-21,24-28]$. Some properties of the determinant, minors and spectrum of the resistance matrix can be found in $[3,4,6,22,24,25]$.

It is known that $r_{i j}(G) \leqslant d_{i j}(G)\left(d_{i j}(G)\right.$ denotes the distance between $i$ and $\left.j\right)$, with equality if and only if $i$ and $j$ are connected by a unique path [19]. Hence for a tree $T$, $R_{T}$ is equal to the distance matrix of $T$. The determinant and the inverse of the distance matrix of a tree are given in $[15,16]$. These formulas have been extended to the resistance matrix [3]. In [23], Merris gave an inequality for the spectrum of the distance matrix of a tree. This inequality also holds for the spectrum of the resistance matrix of any connected graph [24].

For a connected graph $G$ of order $n$, let $D_{i}=\sum_{j=1}^{n} d_{i j}(G), R_{i}=\sum_{j=1}^{n} r_{i j}(G)$. If $D_{1}=D_{2}=\cdots=D_{n}$, then $G$ is called transmission-regular $[1,2]$. Similar to transmissionregular graphs, we say that $G$ is resistance-regular if $R_{1}=R_{2}=\cdots=R_{n}$.

In this paper, we show that $R_{G}$ determines the structure of any connected graph $G$ up to isomorphism. The structure of $G$ or the number of spanning trees of $G$ is determined by partial entries of $R_{G}$ under certain conditions. We give some characterizations of resistance-regular graphs and graphs with few distinct R-eigenvalues. Applying properties of the resistance matrix, we obtain a characterization of strongly regular graphs via resistance distance.

\section{Preliminaries}

For a graph $G$, let $A_{G}$ denote the adjacency matrix of $G$, and let $D_{G}$ denote the diagonal matrix of vertex degrees of $G$. The matrix $L_{G}=D_{G}-A_{G}$ is called the Laplacian matrix of $G$.

The $\{1\}$-inverse of a matrix $A$ is a matrix $X$ such that $A X A=A$. If $A$ is singular, then it has infinite many $\{1\}$-inverses $[5,11]$. We use $A^{(1)}$ to denote any $\{1\}$-inverse of $A$. Let $(A)_{u v}$ or $A_{u v}$ denote the $(u, v)$-entry of $A$.

Lemma 1. $[5,11]$ Let $G$ be a connected graph. If $L_{G}^{(1)}$ is a symmetric $\{1\}$-inverse of $L_{G}$, then $r_{u v}(G)=\left(L_{G}^{(1)}\right)_{u u}+\left(L_{G}^{(1)}\right)_{v v}-2\left(L_{G}^{(1)}\right)_{u v}$.

For a real matrix $A$, the Moore-Penrose inverse of $A$ is the unique real matrix $A^{+}$ such that $A A^{+} A=A, A^{+} A A^{+}=A^{+},\left(A A^{+}\right)^{\top}=A A^{+}$and $\left(A^{+} A\right)^{\top}=A^{+} A$. Let $I$ denote the identity matrix, and let $J_{m \times n}$ denote an $m \times n$ all-ones matrix.

Lemma 2. [18] Let $G$ be a connected graph of order $n$. Then $L_{G}^{+} J_{n \times n}=J_{n \times n} L_{G}^{+}=0$, $L_{G} L_{G}^{+}=L_{G}^{+} L_{G}=I-\frac{1}{n} J_{n \times n}$. 
For a vertex $u$ of a graph $G$, let $L_{G}(u)$ denote the principal submatrix of $L_{G}$ obtained by deleting the row and column corresponding to $u$. By the Matrix-Tree Theorem [5], $L_{G}(u)$ is nonsingular if $G$ is connected.

Lemma 3. Let $G$ be a connected graph of order $n$. Then $\left(\begin{array}{cc}L_{G}(u)^{-1} & 0 \\ 0 & 0\end{array}\right) \in \mathbb{R}^{n \times n}$ is a symmetric $\{1\}$-inverse of $L_{G}$, where $u$ is the vertex corresponding to the last row of $L_{G}$.

Proof. Suppose that $L_{G}=\left(\begin{array}{cc}L_{G}(u) & x \\ x^{\top} & d_{u}\end{array}\right)$, where $d_{u}$ is the degree of $u$. Since $G$ is connected, $L_{G}(u)$ is nonsingular. By using the Schur complement formula, we have $\operatorname{rank}\left(L_{G}\right)=\operatorname{rank}\left(L_{G}(u)\right)+\operatorname{rank}\left(d_{u}-x^{\top} L_{G}(u)^{-1} x\right)=n-1$. By $\operatorname{rank}\left(L_{G}(u)\right)=n-1$, we get $d_{u}=x^{\top} L_{G}(u)^{-1} x$. Then

$$
L_{G}\left(\begin{array}{cc}
L_{G}(u)^{-1} & 0 \\
0 & 0
\end{array}\right) L_{G}=\left(\begin{array}{cc}
I & 0 \\
x^{\top} L_{G}(u)^{-1} & 0
\end{array}\right)\left(\begin{array}{cc}
L_{G}(u) & x \\
x^{\top} & d_{u}
\end{array}\right)=L_{G} .
$$

Hence $\left(\begin{array}{cc}L_{G}(u)^{-1} & 0 \\ 0 & 0\end{array}\right)$ is a symmetric $\{1\}$-inverse of $L_{G}$.

Lemma 4. [11] Let $M=\left(\begin{array}{cc}A & B \\ B^{\top} & C\end{array}\right)$ be a nonsingular matrix, and $A$ is nonsingular. Then $M^{-1}=\left(\begin{array}{cc}A^{-1}+A^{-1} B S^{-1} B^{\top} A^{-1} & -A^{-1} B S^{-1} \\ -S^{-1} B^{\top} A^{-1} & S^{-1}\end{array}\right)$, where $S=C-B^{\top} A^{-1} B$.

For a connected graph $G$ of order $n$, let $\tau_{i}=2-\sum_{j \in \Gamma(i)} r_{i j}(G)$, where $\Gamma(i)$ denotes the set of all neighbors of $i$. Let $\tau$ be be the $n \times 1$ vector with components $\tau_{1}, \ldots, \tau_{n}$.

Lemma 5. $[3,5]$ Let $G$ be a connected graph of order $n$, and let $X=\left(L_{G}+\frac{1}{n} J_{n \times n}\right)^{-1}$, $\widetilde{X}=\operatorname{diag}\left(X_{11}, \ldots, X_{n n}\right)$. Then the following hold:

(a) $\tau=L_{G} \widetilde{X} \mathbf{j}+\frac{2}{n} \mathbf{j}$, where $\mathbf{j}$ is an all-ones column vector.

(b) $R_{G}=\widetilde{X} J_{n \times n}+J_{n \times n} \tilde{X}-2 X$.

(c) $L_{G}^{+}=X-\frac{1}{n} J_{n \times n}$.

For a real symmetric matrix $M$ of order $n$, let $\lambda_{1}(M) \geqslant \lambda_{2}(M) \geqslant \cdots \geqslant \lambda_{n}(M)$ denote the eigenvalues of $M$.

Lemma 6. [24] Let $G$ be a connected graph of order $n$. Then

$$
0>-\frac{2}{\lambda_{1}\left(L_{G}\right)} \geqslant \lambda_{2}\left(R_{G}\right) \geqslant-\frac{2}{\lambda_{2}\left(L_{G}\right)} \geqslant \cdots \geqslant-\frac{2}{\lambda_{n-1}\left(L_{G}\right)} \geqslant \lambda_{n}\left(R_{G}\right) .
$$

The Kirchhoff index of $G$ is defined as $\operatorname{Kf}(G)=\frac{1}{2} \sum_{i=1}^{n} \sum_{j=1}^{n} r_{i j}(G)$.

Lemma 7. [17, 29] Let $G$ be a connected graph of order $n$. Then

$$
\operatorname{Kf}(G)=n \sum_{i=1}^{n-1} \frac{1}{\lambda_{i}\left(L_{G}\right)} .
$$


Lemma 8. [14, 24] Let $G$ be a connected graph of order $n$. Then

$$
\sum_{i j \in E(G)} r_{i j}(G)=n-1
$$

\section{Main results}

All connected graphs in this section have at least two vertices. We first show that the structure of a connected graph is determined by its resistance matrix up to isomorphism, i.e., if two connected graphs have the same resistance matrix, then they are isomorphic.

Theorem 9. For any connected graph $G$, the structure of $G$ is determined by $R_{G}$ up to isomorphism.

Proof. By Lemma 3, the matrix $\left(\begin{array}{cc}L_{G}(u)^{-1} & 0 \\ 0 & 0\end{array}\right)$ is a symmetric $\{1\}$-inverse of $L_{G}$, where $u$ is the vertex corresponding to the last row of $L_{G}$. Since $R_{G}$ is known, by Lemma 1, all entries of $L_{G}(u)^{-1}$ is known, i.e., $L_{G}(u)$ is determined by $R_{G}$. Since each row (column) sum of $L_{G}$ is $0, L_{G}$ is determined by $R_{G}$. Hence $G$ is determined by $R_{G}$ up to isomorphism.

A vertex of degree one is called a pendant vertex. For a vertex $u$ of a connected graph $G$, let $R_{G}(u)$ denote the principal submatrix of $R_{G}$ obtained by deleting the row and column corresponding to $u$. Next we show that $R_{G}(u)$ determines $G$ up to isomorphism if $u$ is not a pendant vertex, i.e., if $u$ is not a pendant vertex of $G$, and $H$ is a connected graph satisfying $R_{H}(v)=R_{G}(u)$ for some $v \in V(H)$, then $H$ is isomorphic to $G$.

Theorem 10. For a connected graph $G$, if $u$ is a vertex of $G$ with degree larger than one, then $R_{G}(u)$ determines $G$ up to isomorphism.

Proof. Without loss of generality, suppose that the first row of $L_{G}$ corresponds to vertex $u$, and the last row of $L_{G}$ corresponds to a vertex $v$. By Lemma $3,\left(\begin{array}{cc}L_{G}(v)^{-1} & 0 \\ 0 & 0\end{array}\right)$ is a symmetric $\{1\}$-inverse of $L_{G}$. Suppose that $L_{G}(v)=\left(\begin{array}{cc}d_{u} & L_{2} \\ L_{2}^{\top} & L_{3}\end{array}\right)$, where $d_{u}$ is the degree of $u$. Let $S=L_{3}-d_{u}^{-1} L_{2}^{\top} L_{2}$. By Lemma 4 , we have

$$
\left(\begin{array}{cc}
L_{G}(v)^{-1} & 0 \\
0 & 0
\end{array}\right)=\left(\begin{array}{ccc}
d_{u}^{-1}+d_{u}^{-2} L_{2} S^{-1} L_{2}^{\top} & -d_{u}^{-1} L_{2} S^{-1} & 0 \\
-d_{u}^{-1} S^{-1} L_{2}^{\top} & S^{-1} & 0 \\
0 & 0 & 0
\end{array}\right)
$$

Since $R_{G}(u)$ is known, by Lemma 1, all entries of $S^{-1}$ are known, i.e., $S$ is determined by $R_{G}(u)$. Since $d_{u}>1$ and $S=L_{3}-d_{u}^{-1} L_{2}^{\top} L_{2}$, the following hold:

(1) For any vertex $i \in V(G) \backslash\{u, v\}, i$ and $u$ are adjacent if $(S)_{i i}$ is not an integer, are non-adjacent if $(S)_{i i}$ is an integer. Moreover, the degree of $i$ is $d_{i}=\left\lceil(S)_{i i}\right\rceil$, where $\left\lceil(S)_{i i}\right\rceil$ is the smallest integer larger than or equal to $(S)_{i i}$. 
(2) There exists a vertex $i \in V(G) \backslash\{u, v\}$ such that $i$ and $u$ are adjacent, and $d_{u}=\left(\left\lceil(S)_{i i}\right\rceil-(S)_{i i}\right)^{-1}$.

(3) For any vertex $i, j \in V(G) \backslash\{u, v\}, i$ and $j$ are adjacent if $(S)_{i j} \leqslant-1$, are nonadjacent if $(S)_{i j}>-1$.

From (1)-(3) we know that $G$ is determined by $S$ up to isomorphism. Since $S$ is determined by $R_{G}(u), R_{G}(u)$ determines $G$ up to isomorphism.

Remark 1. Let $P_{n}$ denote the path with $n$ vertices. Let $G$ be the graph obtained from $P_{n}$ by attaching a pendant vertex $u$ at a vertex of degree two, and let $v$ be a pendant vertex of $P_{n+1}$. In this case, we have $R_{G}(u)=R_{P_{n+1}}(v)$ and $G$ is not isomorphic to $P_{n+1}$. Hence the condition " $u$ is a vertex of degree larger than one" in Theorem 10 is necessary.

Let $t(G)$ denote the number of spanning trees of a graph $G$. If $V_{1}$ and $V_{2}$ are disjoint subsets of $V(G)$, then we define $E\left(V_{1}, V_{2}\right)=\left\{i j \in E(G): i \in V_{1}, j \in V_{2}\right\}$.

Theorem 11. Let $G$ be a connected graph whose vertex set has a partition $V(G)=$ $V_{1} \cup V_{2} \cup\{u\}$, and $G-u$ has a unique perfect matching $M$ satisfying $M \subseteq E\left(V_{1}, V_{2}\right)$. Let $R_{G}=\left(\begin{array}{ccc}R_{1} & R_{3} & a_{1} \\ R_{3}^{\top} & R_{2} & a_{2} \\ a_{1}^{\top} & a_{2}^{\top} & 0\end{array}\right)$, where $R_{1}$ and $R_{2}$ are principal matrices of $R_{G}$ corresponding to $V_{1}$ and $V_{2}$ respectively. Then $t(G)$ is determined by $a_{1}, a_{2}$ and $R_{3}$.

Proof. Without loss of generality, suppose that the last row of $L_{G}$ corresponds to the vertex $u$. By Lemma $3,\left(\begin{array}{cc}L_{G}(u)^{-1} & 0 \\ 0 & 0\end{array}\right)$ is a symmetric $\{1\}$-inverse of $L_{G}$. Since $G-u$ has a unique perfect matching $M$ satisfying $M \subseteq E\left(V_{1}, V_{2}\right), L_{G}(u)$ can be partitioned as $L_{G}(u)=\left(\begin{array}{ll}L_{1} & L_{3} \\ L_{3}^{\top} & L_{2}\end{array}\right)$, where $L_{3}$ is an upper triangular matrix, $L_{1}$ and $L_{2}$ correspond to $V_{1}$ and $V_{2}$ respectively. Let $S=L_{2}-L_{3}^{\top} L_{1}^{-1} L_{3}$. By Lemma 4, we have

$$
\left(\begin{array}{cc}
L_{G}(u)^{-1} & 0 \\
0 & 0
\end{array}\right)=\left(\begin{array}{ccc}
L_{1}^{-1}+L_{1}^{-1} L_{3} S^{-1} L_{3}^{\top} L_{1}^{-1} & -L_{1}^{-1} L_{3} S^{-1} & 0 \\
-S^{-1} L_{3}^{\top} L_{1}^{-1} & S^{-1} & 0 \\
0 & 0 & 0
\end{array}\right)
$$

Since $a_{1}$ and $a_{2}$ are known, by Lemma 1 , all diagonal entries of $L_{G}(u)^{-1}$ are known. Since $R_{3}$ is also known, by Lemma 1 , the matrix $A=-L_{1}^{-1} L_{3} S^{-1}$ is $\operatorname{known}$. Hence $\operatorname{det}(A)=$ $\operatorname{det}\left(-L_{3}\right)\left[\operatorname{det}\left(L_{1}\right) \operatorname{det}(S)\right]^{-1}$ is determined by $a_{1}, a_{2}$ and $R_{3}$. Note that $-L_{3}$ is an upper triangular matrix and each diagonal entry of $-L_{3}$ is 1 . So $\operatorname{det}(A)=\left[\operatorname{det}\left(L_{1}\right) \operatorname{det}(S)\right]^{-1}$. From the Matrix-Tree Theorem, we have $t(G)=\operatorname{det}\left(L_{G}(u)\right)=\operatorname{det}\left(L_{1}\right) \operatorname{det}(S)$. Hence $t(G)$ is determined by $a_{1}, a_{2}$ and $R_{3}$.

Theorem 12. Let $G$ be a connected graph with $n$ vertices. Then the following are equivalent:

(1) $G$ is resistance-regular.

(2) The spectral radius of $R_{G}$ is

$$
\lambda_{1}\left(R_{G}\right)=\frac{2 \mathrm{Kf}(G)}{n} .
$$


(3) The spectrum of $R_{G}$ is

$$
\lambda_{1}\left(R_{G}\right)=\frac{2 \operatorname{Kf}(G)}{n}, \lambda_{i}\left(R_{G}\right)=-\frac{2}{\lambda_{i-1}\left(L_{G}\right)}, i=2, \ldots, n .
$$

(4) $X_{11}=\cdots=X_{n n}$, where $X=\left(L_{G}+\frac{1}{n} J_{n \times n}\right)^{-1}$.

(5) $\left(L_{G}^{+}\right)_{11}=\cdots=\left(L_{G}^{+}\right)_{n n}$.

(6) For each $i \in V(G)$, we have $\sum_{j \in \Gamma(i)} r_{i j}(G)=2-\frac{2}{n}$, where $\Gamma(i)$ denotes the set of all neighbors of $i$.

Proof. By [22, Corollary 2.2], we have $(1) \Longleftrightarrow(2)$.

$(2) \Longrightarrow(3)$. The trace of $R_{G}$ is

$$
\sum_{i=1}^{n} \lambda_{i}\left(R_{G}\right)=\frac{2 \mathrm{Kf}(G)}{n}+\sum_{i=2}^{n} \lambda_{i}\left(R_{G}\right)=0 .
$$

By Lemmas 6 and 7 , we have $\lambda_{i}\left(R_{G}\right)=-\frac{2}{\lambda_{i-1}\left(L_{G}\right)}, i=2, \ldots, n$.

$(3) \Longrightarrow(2)$. Obviously.

$(1) \Longleftrightarrow(4)$. By part (b) of Lemma 5 , we have $R_{G} \mathbf{j}=n \tilde{X} \mathbf{j}+\left(\sum_{i=1}^{n} X_{i i}\right) \mathbf{j}-2 \mathbf{j}$, where $\mathbf{j}$ is an all-ones column vector. Hence $G$ is resistance-regular if and only if $X_{11}=\cdots=X_{n n}$, where $X=\left(L_{G}+\frac{1}{n} J_{n \times n}\right)^{-1}$.

By part (c) of Lemma 5 , we have $(4) \Longleftrightarrow(5)$.

$(4) \Longleftrightarrow(6)$. By Lemma 5(a), (4) is equivalent to $\tau=\frac{2}{n} \mathbf{j}$; that is, $\sum_{j \in \Gamma(i)} r_{i j}(G)=2-\frac{2}{n}$ for any $i \in V(G)$.

Remark 2. For any nonsingular matrix $B$, there exists polynomial $p(x)$ such that $B^{-1}=$ $p(B)$ [7]. Hence $X=\left(L_{G}+\frac{1}{n} J_{n \times n}\right)^{-1}$ is a polynomial in $L_{G}+\frac{1}{n} J_{n \times n}$. If $G$ is a connected regular graph of degree $r$, then $J_{n \times n}$ is a polynomial in $A_{G}$ (see [5, Theorem 6.12]). In this case, $X=\left(r I-A_{G}+\frac{1}{n} J_{n \times n}\right)^{-1}$ is a polynomial in $A_{G}$. A graph $G$ of order $n$ is called walk-regular, if $\left(A_{G}^{k}\right)_{11}=\cdots=\left(A_{G}^{k}\right)_{n n}$ for any $k \geqslant 0$ [10]. For a connected walkregular graph $G$ of degree $r$, since $X=\left(r I-A_{G}+\frac{1}{n} J_{n \times n}\right)^{-1}$ is a polynomial in $A_{G}$ and $\left(A_{G}^{k}\right)_{11}=\cdots=\left(A_{G}^{k}\right)_{n n}$ for any $k \geqslant 0$, we have $X_{11}=\cdots=X_{n n}$. By Theorem 12, connected walk-regular graphs (including distance-regular graphs and vertex-transitive graphs) are resistance-regular.

Graphs with few distinct eigenvalues with respect to adjacency matrix and Laplacian matrix have interesting combinatorial properties $[10,13]$. Next we consider graphs with few distinct R-eigenvalues.

Theorem 13. A connected graph with two distinct $R$-eigenvalues is a complete graph.

Proof. Let $G$ be a connected graph of order $n$ with two distinct R-eigenvalues $\lambda_{1}>\lambda_{2}$. Since $R_{G}$ is irreducible and nonnegative, $\lambda_{1}$ is simple. So $R_{G}-\lambda_{2} I$ has rank 1 . Since each diagonal entry of $R_{G}$ is 0 , we have $R_{G}-\lambda_{2} I=-\lambda_{2} J_{n \times n}, R_{G}=\lambda_{2}\left(I-J_{n \times n}\right)$. Hence $G$ is resistance-regular. By part (3) of Theorem 12, $L_{G}$ has only one nonzero eigenvalue. So $G$ is complete. 
A strongly regular graph with parameters $(n, k, \lambda, \mu)$ is a $k$-regular graph on $n$ vertices such that for every pair of adjacent vertices there are $\lambda$ vertices adjacent to both, and for every pair of non-adjacent vertices there are $\mu$ vertices adjacent to both. It is well known that a connected regular graph whose adjacency matrix has three distinct eigenvalues is strongly regular [10].

Theorem 14. A resistance-regular graph with three distinct $R$-eigenvalues is strongly regular.

Proof. Let $G$ be a resistance-regular graph of order $n$ with three distinct R-eigenvalues. By part (3) of Theorem 12, $L_{G}$ has two distinct nonzero eigenvalues. Let $\mu_{1}>\mu_{2}>0$ be two distinct nonzero eigenvalues of $L_{G}$. Since $\left(L_{G}-\mu_{1} I\right)\left(L_{G}-\mu_{2} I\right)$ has rank 1 and row sum $\mu_{1} \mu_{2}$, we have

$$
\begin{aligned}
& \left(L_{G}-\mu_{1} I\right)\left(L_{G}-\mu_{2} I\right)=\frac{\mu_{1} \mu_{2}}{n} J_{n \times n}, \\
& L_{G}^{2}-\left(\mu_{1}+\mu_{2}\right) L_{G}+\mu_{1} \mu_{2} I=\frac{\mu_{1} \mu_{2}}{n} J_{n \times n} .
\end{aligned}
$$

By Lemma 2, we have

$$
L_{G} L_{G}^{+}=I-\frac{1}{n} J_{n \times n}, L_{G}^{2} L_{G}^{+}=L_{G}\left(I-\frac{1}{n} J_{n \times n}\right)=L_{G}, \quad J_{n \times n} L_{G}^{+}=0 .
$$

We multiply $L_{G}^{+}$on both side of (3.1), then

$$
\begin{array}{r}
{\left[L_{G}^{2}-\left(\mu_{1}+\mu_{2}\right) L_{G}+\mu_{1} \mu_{2} I\right] L_{G}^{+}=\frac{\mu_{1} \mu_{2}}{n} J_{n \times n} L_{G}^{+},} \\
L_{G}-\left(\mu_{1}+\mu_{2}\right)\left(I-\frac{1}{n} J_{n \times n}\right)+\mu_{1} \mu_{2} L_{G}^{+}=0 .
\end{array}
$$

From part (5) of Theorem 12, we know that $G$ is regular. Since $G$ is a connected regular graph and $L_{G}$ has two distinct nonzero eigenvalues, $G$ is strongly regular.

Theorem 15. Let $G$ be a connected regular graph with diameter at least 2 . Then $G$ is strongly regular if and only if there exist $c_{1}, c_{2}$ such that $r_{i j}(G)=c_{1}$ for any adjacent vertices $i, j \in V(G)$, and $r_{i j}(G)=c_{2}$ for any non-adjacent vertices $i, j \in V(G)$.

Proof. Suppose that $G$ has $n$ vertices and $m$ edges. We need to prove that $G$ is strongly regular if and only if there exist $c_{1}, c_{2}$ such that

$$
R_{G}=c_{1} A_{G}+c_{2}\left(J_{n \times n}-I-A_{G}\right) .
$$

If $G$ is strongly regular, then $r_{i j}(G)$ depends only on the distance between $i$ and $j$ (see $[8,20])$, i.e., the equation (3.2) holds.

If (3.2) holds, then by Lemma 8, we have $c_{1}=\frac{n-1}{m}$. Then $\sum_{j \in \Gamma(i)} r_{i j}(G)=\frac{(n-1) k}{m}=$ $2-\frac{2}{n}$ for each $i \in V(G)$, where $k$ is the degree of regular graph $G$. By parts (4) and (6) of 
Theorem 12, there exists $c_{0}$ such that $c_{0}=X_{11}=\cdots=X_{n n}$, where $X=\left(L_{G}+\frac{1}{n} J_{n \times n}\right)^{-1}=$ $\left(k I+\frac{1}{n} J_{n \times n}-A_{G}\right)^{-1}$. By part (b) of Lemma 5 and (3.2), we have

$$
\begin{gathered}
R_{G}=2 c_{0} J_{n \times n}-2 X=c_{2}\left(J_{n \times n}-I\right)+\left(c_{1}-c_{2}\right) A_{G}, \\
2 c_{0} J_{n \times n} X^{-1}-2 I=c_{2}\left(J_{n \times n}-I\right) X^{-1}+\left(c_{1}-c_{2}\right) A_{G} X^{-1} .
\end{gathered}
$$

Since $G$ is regular, by the equation (3.3), there exist $a_{1}, a_{2}, a_{3}$ such that

$$
\left(c_{1}-c_{2}\right) A_{G}^{2}+a_{1} A_{G}=a_{2} I+a_{3} J_{n \times n} .
$$

If $c_{1}=c_{2}$, then by (3.2), we get $R_{G}=c_{1}\left(J_{n \times n}-I\right)$. In this case, $R_{G}$ has two distinct eigenvalues. By Theorem 3.5, $G$ is complete, a contradiction to that the diameter of $G$ at least 2. Hence $c_{1} \neq c_{2}$. By the equation (3.4), we know that there exist $\lambda, \mu$ such that $\left(A_{G}^{2}\right)_{i j}=\lambda$ for any adjacent vertices $i, j \in V(G)$, and $\left(A_{G}^{2}\right)_{i j}=\mu$ for any non-adjacent vertices $i, j \in V(G)$. Then $G$ is a strongly regular graph with parameters $(n, k, \lambda, \mu)$.

\section{Concluding remarks}

In this paper, the relationship between the graph structure and resistance matrix is studied, and some spectral properties of the resistance matrix are obtained. We list some problems as follows.

(1) For a connected graph $G$, the structure of $G$ or $t(G)$ is determined by partial entries of $R_{G}$ under certain conditions (see Theorems 10 and 11). Are there some other graph properties can be determined by partial entries of the resistance matrix?

(2) Some equivalent conditions for resistance-regular graphs are given in Theorem 12. From Remark 2, we know that connected walk-regular graphs are resistance-regular. It is natural to consider the problem "Which graphs are resistance-regular?". Note that a transmission-regular graph does not need to be a (degree) regular graph [1, 2]. Is there a nonregular resistance-regular graph?

\section{Acknowledgements}

This work is supported by the Natural Science Foundation of the Heilongjiang Province (No. QC2014C001), the National Natural Science Foundation of China (No. 11371109), and the Fundamental Research Funds for the Central Universities.

\section{References}

[1] M. Aouchiche and P. Hansen. On a conjecture about the Szeged index. European J. Combin., 31:1662-1666, 2010.

[2] M. Aouchiche and P. Hansen. Two Laplacians for the distance matrix of a graph. Linear Algebra Appl., 439:21-33, 2013. 
[3] R.B. Bapat. Resistance matrix of a weighted graph. MATCH Commun. Math. Comput. Chem., 50:73-82, 2004.

[4] R.B. Bapat. Resistance matrix and q-Laplacian of a unicyclic graph. Ramanujan Math. Soc. Lect. Notes Ser., 7:63-72, 2008.

[5] R.B. Bapat. Graphs and Matrices. Springer, London, 2010.

[6] R.B. Bapat and S. Sivasubramanian. Identities for minors of the Laplacian, resistance and distance matrices. Linear Algebra Appl., 435:1479-1489, 2011.

[7] A. Ben-Israel and T.N.E. Greville. Generalized Inverses: Theory and Applications. Springer, New York, 2003.

[8] N. Biggs. Potential theory on distance-regular graphs. Combin. Probab. Comput., 2:243-255, 1993.

[9] B. Bollobás and G. Brightwell. Random walks and electrical resistances in products of graphs. Discrete Appl. Math., 73:69-79, 1997.

[10] A.E. Brouwer and W.H. Haemers. Spectra of Graphs. Springer, New York, 2012.

[11] C. Bu, B. Yan, X. Zhou and J. Zhou. Resistance distance in subdivision-vertex join and subdivision-edge join of graphs. Linear Algebra Appl., 458:454-462, 2014.

[12] H. Chen and F.J. Zhang. Resistance distance and the normalized Laplacian spectrum. Discrete Appl. Math., 155:654-661, 2007.

[13] E.R. van Dam and W.H. Haemers. Graphs with constant $\mu$ and $\bar{\mu}$. Discrete Math., 182:293-307, 1998.

[14] R.M. Foster. The average impedance of an electrical network. In Contributions to Applied Mechanics, pages 333-340. Ann Arbor, Michigan, 1949.

[15] R.L. Graham and L. Lovász. Distance matrix polynomials of trees. Adv. Math., 29:60-88, 1978.

[16] R.L. Graham and H.O. Pollack. On the addressing problem for loop switching. Bell Syst. Tech. J., 50:2495-2519, 1971.

[17] I. Gutman and B. Mohar. The quasi-Wiener and the Kirchhoff indices coincide. J. Chem. Inf. Comput. Sci., 36:982-985, 1996.

[18] I. Gutman and W.J. Xiao. Generalized inverse of the Laplacian matrix and some applications. Bull. Acad. Serbe Sci. Arts (Cl. Sci. Math. Natur.), 129:15-23, 2004.

[19] D.J. Klein and M. Randić. Resistance distance. J. Math. Chem., 12:81-95, 1993.

[20] J.H. Koolen, G. Markowsky and J. Park. On electric resistances for distance-regular graphs. European J. Combin., 34:770-786, 2013.

[21] X. Liu, J. Zhou and C. Bu. Resistance distance and Kirchhoff index of R-vertex join and R-edge join of two graphs. Discrete Appl. Math., 187:130-139, 2015.

[22] A.D.G. Maden, I. Gutman and A.S. Çevic. Bounds for resistance distance spectral radius. Hacet. J. Math. Stat., 42:43-50, 2013.

[23] R. Merris. The distance spectrum of a tree. J. Graph Theory, 14:365-369, 1990. 
[24] L. Sun, W. Wang, J. Zhou and C. Bu. Some results on resistance distances and resistance matrices. Linear and Multilinear Algebra, 63:523-533, 2015.

[25] W.J. Xiao and I. Gutman. Resistance distance and Laplacian spectrum. Theor. Chem. Acc., 110:284-289, 2003.

[26] Y.J. Yang and D.J. Klein. Resistance distance-based graph invariants of subdivisions and triangulations of graphs. Discrete Appl. Math., 181:260-274, 2015.

[27] Y.J. Yang and H.P. Zhang. Some rules on resistance distance with applications. J. Phys. A: Math. Theor., 41:445203, 2008.

[28] B. Zhou and N. Trinajstić. On resistance-distance and Kirchhoff index. J. Math. Chem., 46:283-289, 2009.

[29] H.Y. Zhu, D.J. Klein and I. Lukovits. Extensions of the Wiener number. J. Chem. Inf. Comput. Sci., 36:420-428, 1996. 